\title{
Discrete Wavelet Transform with Eigenface to Enhance Face Recognition Rate
}

\author{
Shakir Fattah Kak ${ }^{1}$ Firas Mahmood Mustafa ${ }^{2}$ Pedro R. Valente ${ }^{3}$ \\ ${ }^{1}$ Duhok Polytechnic University (DPU) - Akre Technical Institute, Kurdistan Region - Iraq \\ ${ }^{2}$ Department Computer and Communications Engineering, Nawroz University, Duhok, Kurdistan Region - Iraq \\ ${ }^{3}$ Portucalense University (UPT) , Portugal
}

\begin{abstract}
In a recent past, face recognition was one of the most popular methods and successful application of image processing field which is widely used in security and biometric applications. The innovation of new approaches to face identification technologies is continuously subject to building much strong face recognition algorithms. Face recognition in real-time applications has been fast-growing challenging and interesting. The human face identification process is not trivial task especially different face lighting and poses are captured to be matched. In this study, the proposed method is tested using a benchmark ORL database that contains 400 images of 40 persons as the variant posse, lighting, etc. Discrete avelet Transform technique is applied on the ORL database to enhance the accuracy and the recognition rate. The best recognition rate result obtained is $99.25 \%$, when tested using 9 training images and 1 testing image with cosine distance measurement. The recognition rate Increased when applying 2-level of DWT with the bior5.5 filter on training image database and the test image. For feature extraction and dimension reduction, PCA is used. Euclidean distance, Manhattan distance, and Cosine distance are Distance measures used for the matching process.
\end{abstract}

KEYWORDS : DWT, ORL Database, Face Recognition, PCA, Distance measures.

\section{INTRODUCTION}

Recognize individuals, using facial feature, is one of biometrics' most popular method, entitled as recognition schema. Also, it is considered as a biometrics technology that has been adopted in the technology industry and research community. Researchers focus in this area in the last few decades. Thus because, for its potential applications in the areas such as personal identification and authentication, digital libraries, surveillance systems, security access control, border control, military, e-commerce and human-computer interaction (Abdulrahman, Dambatta, Muhammad, \& Muhammad, 2014); (Bagherian \& Rahmat, 2008). This method uses facial details from

Academic Journal of Nawroz University

(AJNU) Volume 7, No 4 (2018).

Regular research paper : Published 8 December 2018

Corresponding author's e-mail : sali29870@gmail.com

Copyright (C2017 Shakir Fattah Kak1, Firas Mahmood

Mustafa $^{2}$, Pedro R. Valente ${ }^{3 .}$

This is an open access article distributed under the Creative

Commons Attribution License. captured by digital images to verify and identify the person recognizing faces from frontal view and normal illuminating conditions are a reasonable well-solved problem. However, the difficulty arises when the face is under the influence of abnormal conditions such as face expression, position. In computer Machine, algorithms are used the information content extracted from the face and verify or identify it as known or unknown by comparing with the database it seems to be very difficult and faces many challenges, for example, to recognize the face from a general point under different illumination conditions and facial expressions such as smile, cry sad, emotion etc. however, human very easily can recognize facial expressions and different faces (Abdulrahman, Dambatta, Muhammad, \& Muhammad, 2014); (Setyadi, Harsono, \& Wasista, 2015); (Gumede, Viriri, \& Gwetu, 2017). Extracting features in transforms are based on a local operator, measurements, and statistical analysis. The greater part of the methodologies of extractor depends on wavelets. This can be achieved by dilations and rotations. In face recognition process, DWT technique is an extremely mainstream technique to recognize the face images 
from the database. One of the special kinds of the function is wavelet, which is used for a short period of time. In DWT image can be divided into approximation, vertical, horizontal, and diagonal features (Rao, Swamy, \& sheela, 2012). Face recognition technique can be defined as given a face image captured by digital camera and verify or identify the input face by finding the best similar face stored in a database. This means that an input face can be classified either unknown or known, after matching it with labeled person image kept in a database (Abdulrahman, Dambatta, Muhammad, \& Muhammad, 2014). This research focuses specifically on hybrid methods and featurebased which utilize representations of local face components or fragments. The work described in this paper aims to automatically select the DWT coefficients which contribute most to classification, using both LL and non-LL quadrant features. It then optimizes the feature vector, choosing only the features that are most discriminative. This eliminates the need to overly crop the face images. In this work, proposed a technique to improve face recognition rate and investigates the effectiveness of component-based facial recognition. This research is paper has five sections organized as follow: Section one is an introduction. Section two is dedicated to a literature review related to face recognition. In Section three, the research methodology design is presented by details. Then,, the result and discussion are described in Section Four. Finally, in Section five, the conclusion is presented followed by the list of references.

\section{RELEATED WORK}

The study by (Haider \& Arasi, 2013), a comparison of different features extraction algorithms is done. In this paper, three different features extraction algorithms (Principal Component Analysis (PCA), Discrete Cosine Transform (DCT) and Discrete Wavelet Transform (DWT)) are compared by using Euclidean Distance, Cosine distance and Correlation distance measures where these methods are tested on ORL and Yale Face Databases. The results of face-recognition experiments on ORL database led to improved results, which is nearly $95.33 \%$ recognition rate. The Euclidean Distance is applied after implementing the DWT $3^{\text {rd }}$ level decomposition and the better results obtained when using the PCA model with Cosine distance and Correlation distance. Another publication that test implementation of Face Recognition operations is written by (Abdulrahman, Dambatta, Muhammad, \& Muhammad, 2014), the author's proposed face recognition system that tested on Yale database and MATLAB software. Firstly, the database images are decomposed using a 3-level DWT to reduce dimensionality, finally, the PCA algorithm is implemented as additional dimensionality reduction. In the Eigenface, the weight of a face of the test image is compared with the Eigen face of the database to reconstruct the final face. The better and the closer the reconstructed image to the original image depending on the number of Eigen face used in the reconstruction process. While, a work was done by (Barnouti, 2016) which ORL database has been used to increase the recognition rate of test images. This method that the author used to improve the results is to enter the images into preprocessing operations before the feature extraction stage where the PCA model is used. The results obtained from this work increased the recognition rate to be $97.5 \%$ when tested using 9 training images and 1 testing image. The preprocessing includes increasing database images brightness and resizing the images using 0.3 scales are applied.

\section{METHODOLOGY}

The proposed technique generally designed for face recognition uses 2D face images with different variations in pose, aging, light, and expression. The ORL face images database mostly used in the recognition process, the database contains 400 images of 40 persons, 10 for each one. An individual has 10 different images showing different copies of that person with different positions. The flowchart of the proposed algorithm showed in Fig. 1. 


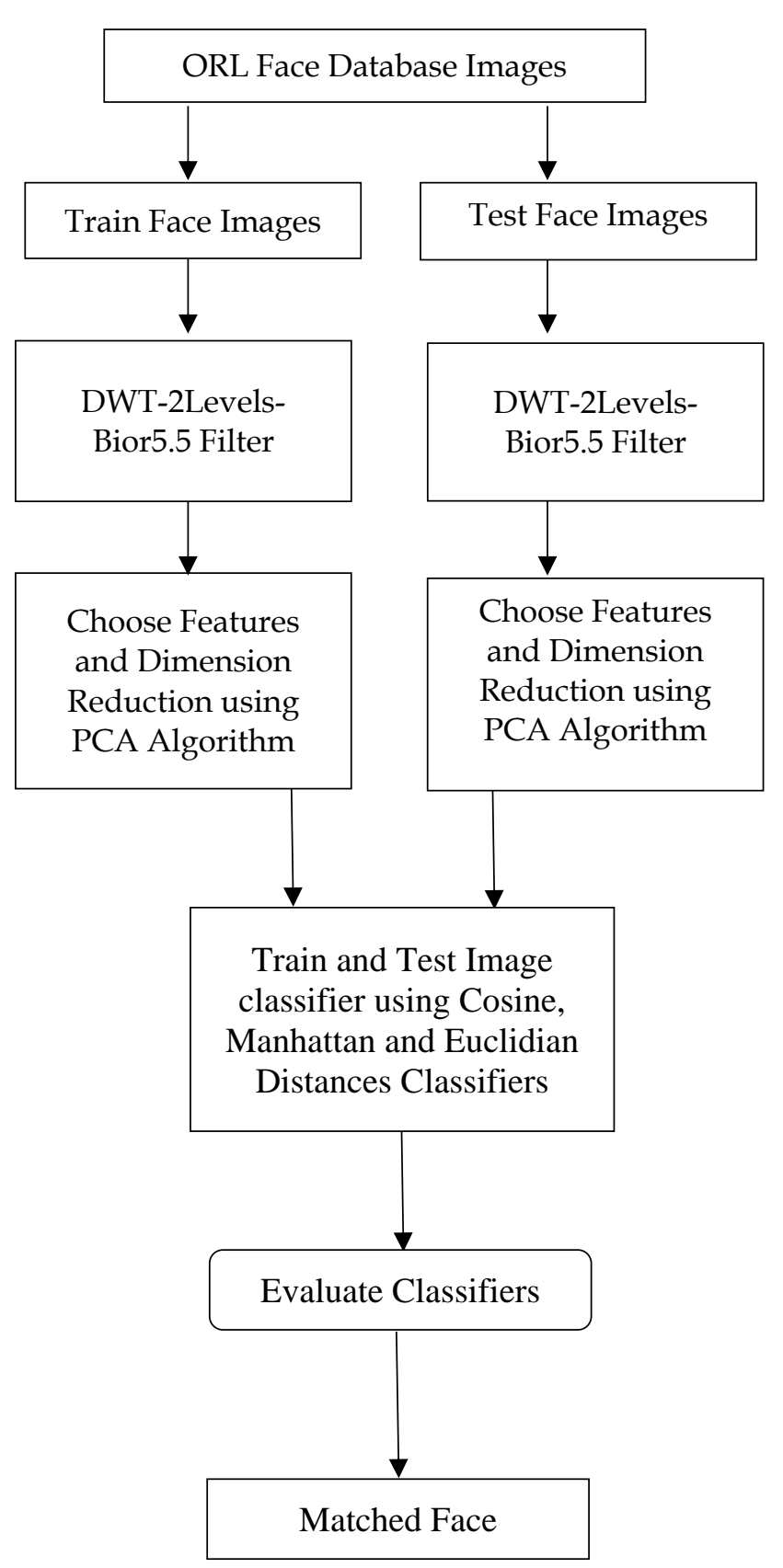

Fig (1) : Flowchart of the proposed technique

The modification proposed is to modify the train and test images in size, then decompose the images with the DWT2 at different levels as a preparing step before feature extraction stage which is done by using PCA scheme. Only the approximation low band (LL) in after the first level of decomposition and the next levels, that removes an image details bands (HL, LH, and $\mathrm{HH}$ ) which supposed represent the unwanted noise of that image. The obtained 2-dimensions images would have decreased in size to $1 / 4$ of the original decomposed image size at each level of DWT decomposition. Fig. 2 shows applying DWT-2-levels on person image in ORL database.

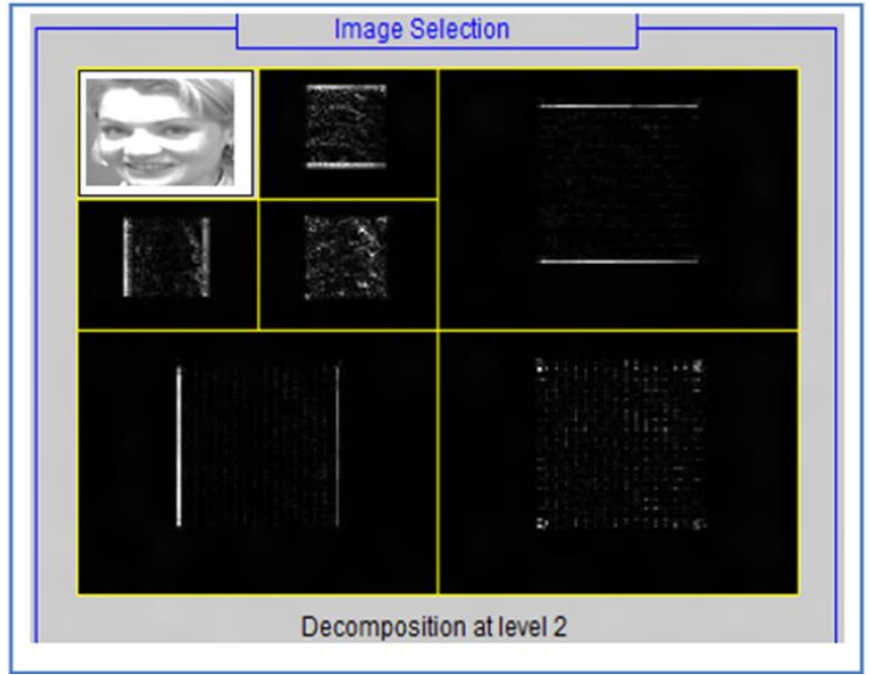

Fig (2) : DWT of the face image at two levels of decomposition

PCA is a statistical projection technique used for face recognition and dimensionality reduction, which improves main recognition problems such as illumination and pose. The images are processed by dividing a person's ten images into two groups test images and train images. The PCA algorithm is implemented one each group at the feature extraction stage. At the first iteration, for the first indexed person, the first image in the test group as compared to 9 images in the training group for that person. At the second iteration, the first two indexed images are compared to the images from the third to the $10^{\text {th }}$ image of the same first indexed person. This process is continued until all the images processed at the $9^{\text {th }}$ iteration for that person, with the first 9 images and the last $10^{\text {th }}$ indexed image of the first person. Similarly, the recognition process is performed to all the remained 39person images in the ORL previously decomposed database images. Distance Classifier is exploited in comparing two different feature extraction methods; two different images are similar when the distance between them is minimal. In this approach, the comparison is made between the original PCA method and the new proposed method DWTPCA. Three different classifiers are used in this issue, Euclidean Distance, City Block Distance and Cosine Distance measurements. The best results of the proposed methods are obtained when using two levels of DWT2 decomposition levels with bior5.5 filters by using cosine distance measurement. The performances of face recognition rate before and after applying the proposed technique are shown in Table2.

\subsection{Principal component analysis}

The Principal Component Analysis (PCA) algorithm is a dimensional-reduction technique. It regards data as 
points in a high-dimensional space, operating by finding a new coordinate system for a dataset, with the axes (or principal components) ordered by the degree of variance contained within the training data. A set of faces can be represented as points in this new coordinate system (Kurmi, Agrawal, \& Baghel, 2014). This method evaluates each one of pixels independently, means, it does not model relationships between neighboring image pixels. Consequently, however, a face image $A$ of dimension $I x j$ is converted to a column vector of length $H$ as in (1) where $\mathrm{H}=I J$.

$$
a=\left[\begin{array}{c}
k_{1.1} \\
k_{1.2} \\
\cdot \\
\cdot \\
k_{1 . j} \\
k_{2.1} \\
\vdots \\
k_{i . j-1} \\
k_{i . j}
\end{array}\right]
$$

The $k_{i . j}$ represents pixel from the $i^{\text {th }}$ row and the $j^{\text {th }}$ column. A set of $W$ face images $\left\{a_{i}\right\}$ may be represented as a matrix $A$ of dimension $N^{*} W$ as in (2).

$A=\left[a_{1} a_{2} a_{3 . . .} a_{W}\right]$

Next, finding the $A^{\prime}$ (the average face) which is subtracted and calculated from each face in $A$ as in (3).

$A^{\prime}=\left[\left(a_{1}-\bar{a}\right)\left(a_{2}-\bar{a}\right)\left(a_{3}-\bar{a}\right) \ldots\left(a_{W}-\bar{a}\right)\right] \quad(3)$

Finally, finding the principal component of this set by computing the eigenvectors of the covariance matrix $C$, as in (4).

$$
C=\sum_{i=1}^{W} A^{\prime} A^{\prime T}
$$

The training set faces are represented by the obtained eigenvectors which are used as an orthogonal basis in this process (Nicholl, Ahmad, \& Amira, 2010).

\subsection{Discrete wavelet transforms}

Transformation a signal is just another form of representing this signal. Transforming from the time domain to frequency domain does not change the information content present in the signal. In most Digital Signal Processing (DSP) applications, the frequency contents of the signal are very important. Wavelet transform provides a compact multi-resolution representation of the image. Wavelet Transform is used to convert a spatial domain image into a frequency domain. It provides the time-frequency representation. The Discrete Wavelet Transform (DWT) consists of multiscale frequency decomposition of an image. In case of DWT for an image, the image is first decomposed into four parts of high, middle and low frequencies LL1, LH1, HL1, HH1 by subsampling horizontally and vertically channels using sub band filter (Mei, Bo, Hua, \& Wei, 2010). There are a lot of Wavelet filters like bior, Daubechies Wavelets, Haar, Coiflets, biorthogonal wavelets, and Symlets. These various transforms are different in mathematical properties such as symmetry, number of vanishing moments and orthogonality. 2D-DWT is implemented as a set of filter banks, comprising of a cascaded scheme of high-pass and low-pass filters. The sub bands labeled LH1, HL1, and HH1 represent the finest scale Wavelet coefficients and LL1 stands for the coarse scale wavelet coefficients (Woods, 2012).

a. LL is a coarser approximation to the original image containing the overall information about the whole image. It is obtained by applying the low-pass filter on both $\mathrm{x}$ and $\mathrm{y}$ coordinates.

b. HL and LH are obtained by applying the high-pass filter on one coordinate and the low-pass filter on the other coordinate.

c. HH shows the high-frequency component of the image in the diagonal direction. It is obtained by applying the high-pass filter on both $\mathrm{x}$ and $\mathrm{y}$ coordinates.

To obtain the next coarser scaled wavelet coefficients, the sub band LL1 is further decomposed and subsampled. This process is repeated several times, which is determined by the requirement of the user, as shown in Fig. 3. 


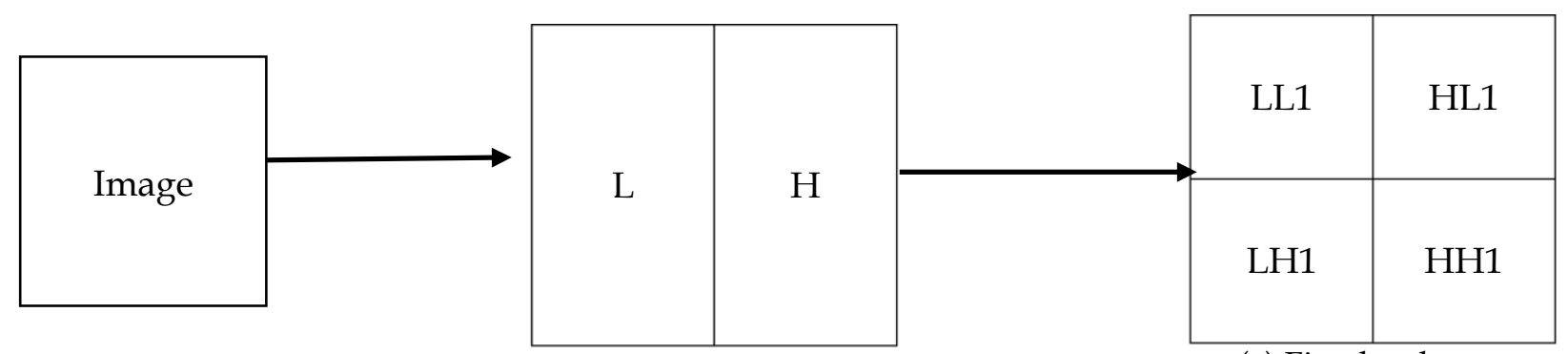

(a) First level

Decomposition

\begin{tabular}{|c|c|c|c|c|c|c|}
\hline & & \multirow{3}{*}{ HL1 } & LL3 & & & \multirow{3}{*}{ HL1 } \\
\hline LL2 & HL2 & & LH3 & $\mathrm{HH} 3$ & HLL & \\
\hline LH2 & $\mathrm{HH} 2$ & & 녀: & & Hн2 & \\
\hline \multicolumn{2}{|c|}{ LH1 } & HH1 & \multicolumn{3}{|c|}{ LH1 } & HH1 \\
\hline
\end{tabular}

(b) Second level decomposition

(c) Third level decomposition

Fig (3) : Multiscale decomposing of an image.

\subsection{Euclidean distance}

The similarity level between input test image and training samples can be classified and obtained by the Euclidean Distance classifier (ED), it is a common method for many distance measurements because it is simple and faster compared with other matching measurements. This method defined as the straight-line distance between two points, which examines the root of square differences between the coordinates of a pair of images. The equation used in the Euclidean distance in order to obtain the differences between training images and test image defined as in (5):

$D(x . y)=\sqrt{\sum_{i=0}^{n}\left(x_{i}-y_{i}\right)^{2}}$

Suppose $\mathrm{x}$ is a test image and $\mathrm{y}$ is a training image where $\mathrm{n}$ is the number of images. Then, Euclidean Distance is computed for all images in training with the test image. A minimum Euclidean Distance classifier is used as a condition in order to find the best-matched test image in the traini

ng samples (Barnouti, Mahmood, \& Matti, 2016)

\subsection{City block distance}

This method is also known as Manhattan Distance (MD) classifier. The sum of absolute differences between two vectors is called the L1 distance, or city-block distance. It follows the triangle inequality process. The reason why it is called the city-block distance, because it represents the distance between points in a city road grid, while the Euclidean straight-line distance. It examines the absolute differences between the coordinates of a pair of objects for any two given vectors $x$ and $y$ as in (6) (Sodhi \& Lal, 2013) ; (Gawande \& Agrawal, 2014):

City Block $(x . y)=|x-y|=\sum_{i=0}^{\text {No.of Images }}\left|x_{i}-y_{i}\right|$

(6)

3.5 Cosine distance:

The Cosine Distance (CD) classifier is another face matching distance classifier between two vectors (or two images on the Vector Space) this metric is a measurement of orientation and not magnitude. It can be as follows:

For any two given vectors $\mathrm{x}$ and $\mathrm{y}$, the cosine similarity, $\theta$, is represented using a dot product and magnitude as Cosine Similarity as in (7):

$\theta(\mathrm{x}, \mathrm{y})=-\frac{x \cdot y}{|| x|||y| \mid}=-\frac{\sum_{i=1}^{n} x_{i} y_{i}}{\sqrt{\sum_{i=1}^{n} x_{i}^{2} \sum_{i=1}^{n} y_{i=1}^{2}}}$

For face matching, the attribute vectors $\mathrm{x}$ and $\mathrm{y}$ are usually the term frequency vectors of the images. The cosine similarity is a method of normalizing image length during the comparison. The resulting similarity ranges from -1 meaning exactly opposite, to 1 meaning the same, with 0 usually indicating independence, and in-between values indicating intermediate similarity or dissimilarity (Gawande \& Agrawal, 2014).

\subsection{Face database}

Database creation and Face image data obtaining have gotten incredible consideration from computer scientists as of late. The aftereffects of face recognition examine profoundly rely upon the adaptability 
(availability of moderately large person samples) of the database utilized. The accessibility of a database that contains a suitable number of illustrative samples is vital in any pattern recognition study.

In this study, ORL image face database is used to test the performance of the proposed schema. It is containing 40 different persons (subjects) with 10 images for the individual person, with a total of 400 images. The 40 persons are 36 males and 4 females are included in the database. The total pixels of each image in this database are 10,304 pixels (Barnouti N. H., 2016). The ORL database properties illustrated in Table1.

Table (1) : ORL Database Properties.

\begin{tabular}{|c|c|}
\hline Properties & Explanation \\
\hline Purpose & $\begin{array}{r}\text { It is used primarily for face } \\
\text { recognition }\end{array}$ \\
\hline $\begin{array}{c}\text { \# of } \\
\text { Subjects }\end{array}$ & 40 \\
\hline \# of Person & 10 \\
\hline \# of Images & 400 \\
\hline
\end{tabular}

\begin{tabular}{|c|c|}
\hline $\begin{array}{c}\text { Video / } \\
\text { Static }\end{array}$ & Static \\
\hline $\begin{array}{c}\text { Gray / } \\
\text { Color }\end{array}$ & Gray \\
\hline $\begin{array}{c}\text { Image } \\
\text { Format }\end{array}$ & Portable Gray Map $\left(^{*}\right.$.pgm) \\
\hline $\begin{array}{c}\text { Image } \\
\text { Resolution }\end{array}$ & $92^{\star} 112$ \\
\hline $\begin{array}{c}\text { Facial } \\
\text { expression }\end{array}$ & $\begin{array}{c}\text { Three facial expressions: } \\
\text { natural, smiling and closed eye }\end{array}$ \\
\hline $\begin{array}{c}\text { Moderate pose variation (up } \\
\text { and down, quarter profile to } \\
\text { frontal view) }\end{array}$ \\
\hline $\begin{array}{c}\text { Image } \\
\text { Background }\end{array}$ & Dark homogeneous \\
\hline $\begin{array}{c}\text { Face } \\
\text { Accessories }\end{array}$ & Glasses \\
\hline
\end{tabular}

As shown in Fig. 4, some face images of several users in the ORL database are randomly selected. It shows that, the user database in this study has variant illuminations and poses of training samples of each user.
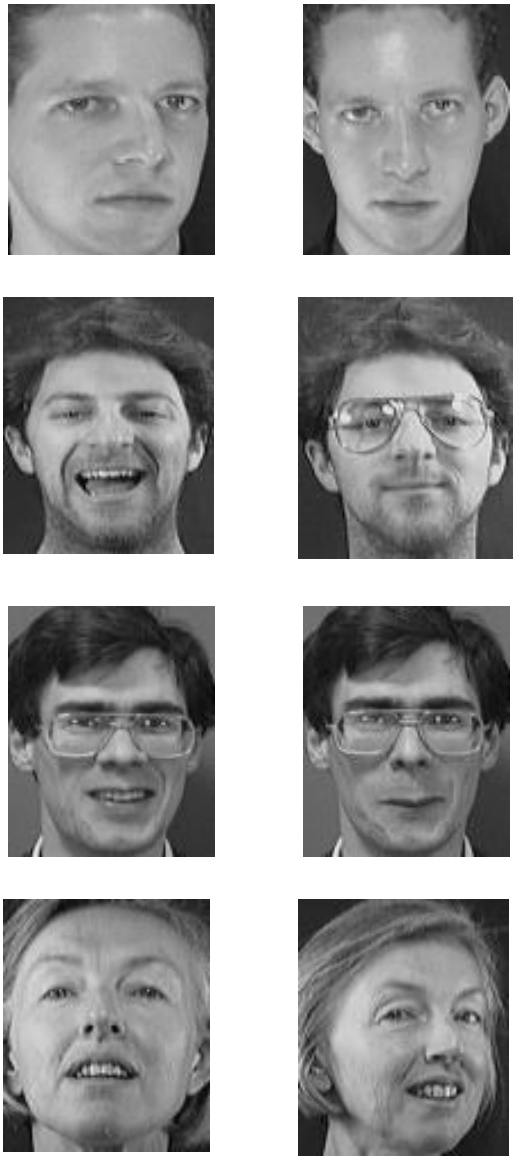
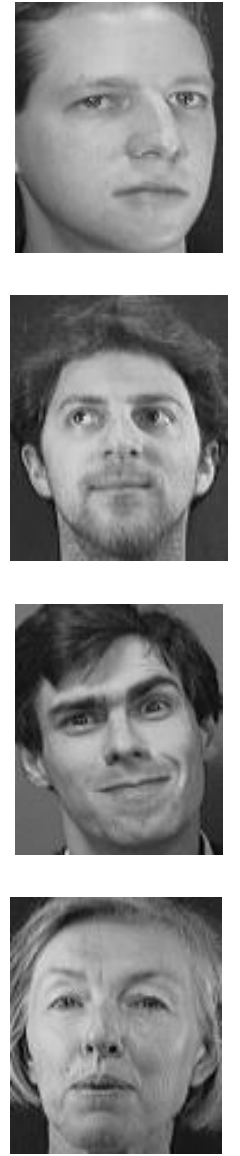
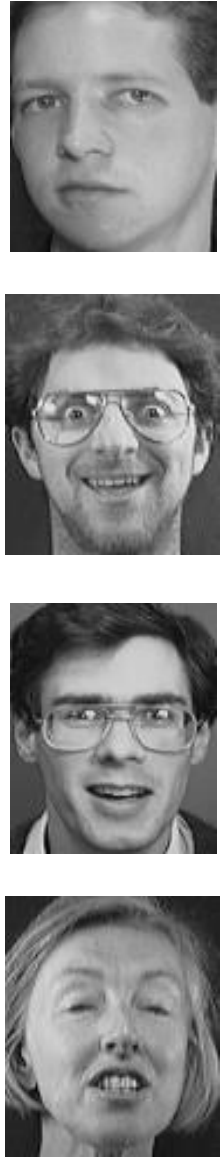
Subject_34
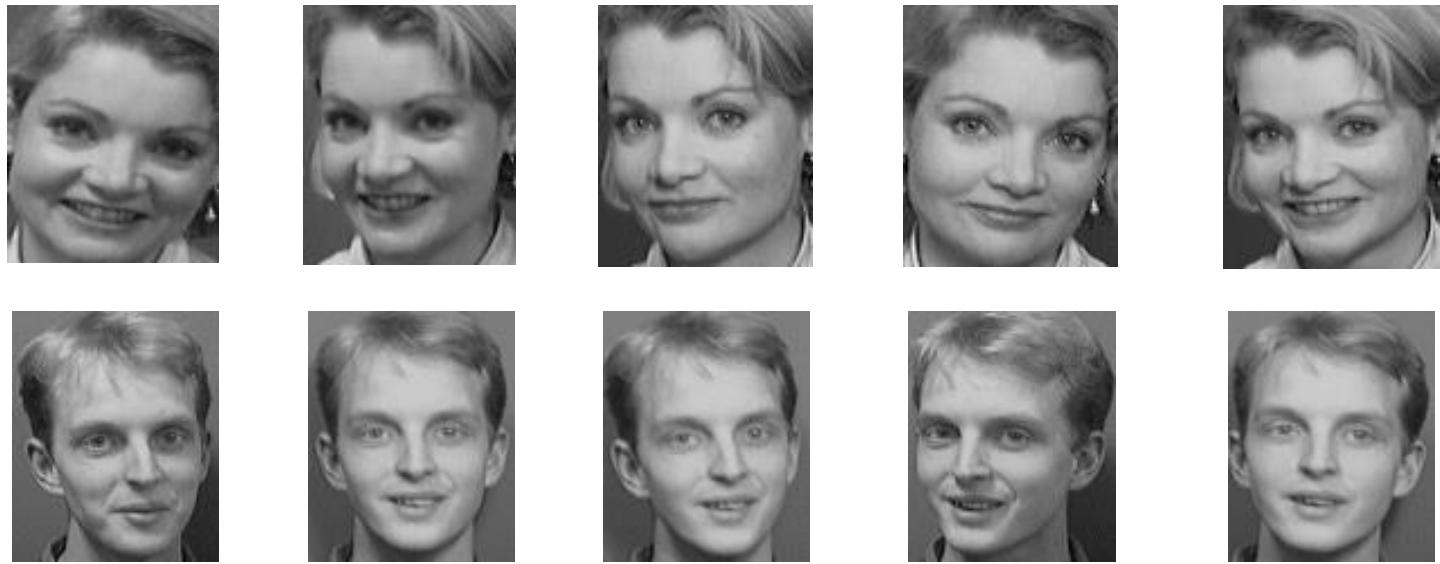

Fig (4) : Face images samples selected randomly form ORL database.

\section{RESULTS AND DISCUSSION}

In this work, recognition rate performance of the presented face recognition schema is tested on ORL face database by showing experiments using MATLAB 2016a. To examine the recognition rate performance, a dissimilar number of testing and training images are used. The ORL database images are chosen in this system that changing in pose, occlusion and illumination intensity since changes in illumination which affects the low-frequency spectrum LL components. DWT method is implemented on the ORL database to increase the recognition rates. These results shows that, when the images of the database were first decomposed by a 2-level DWT using "bior 5.5" wavelet filter before they are next applied the PCA method for final dimensionality reduction, the proposed system DWT(2 levels-bior5.5) with PCA accuracy evaluation on ORL database with distance classifier (Euclidian, cosine and Manhattan) is presented in table2.

Table (2) : Recognition Rate Results of Original PCA and the Proposed System

\begin{tabular}{|c|c|c|c|c|c|c|c|c|}
\hline \multirow[t]{2}{*}{$\begin{array}{c}\text { \# of } \\
\text { Training Images }\end{array}$} & \multirow[t]{2}{*}{$\begin{array}{c}\text { \# of } \\
\text { Testing images }\end{array}$} & \multicolumn{4}{|c|}{ Recognition Rate (Original PCA) } & \multicolumn{3}{|c|}{$\begin{array}{l}\text { Recognition Rate of proposed System } \\
\text { (DWT-2 levels -Bior5.5 filter-PCA) }\end{array}$} \\
\hline & & ED \% & MD \% & CD \% & & ED \% & MD \% & CD \% \\
\hline 1 & 9 & 69.31 & 69.13 & 64.30 & & 76.05 & 76.27 & 73.69 \\
\hline 2 & 8 & 82.50 & 83.125 & 78.53 & & 86.43 & 87.06 & 85.12 \\
\hline 3 & 7 & 88.12 & 88.89 & 84.53 & & 91.67 & 91.96 & 90.89 \\
\hline 4 & 6 & 91.53 & 92.33 & 88.87 & & 94.83 & 95.25 & 94.29 \\
\hline 5 & 5 & 92.85 & 94.35 & 91.35 & & 96.15 & 95.9 & 95.7 \\
\hline 6 & 4 & 93.125 & 95.43 & 93.06 & & 97.18 & 96.87 & 96.62 \\
\hline 7 & 3 & 94.50 & 97.00 & 95.83 & & 97.66 & 97.25 & 97.91 \\
\hline 8 & 2 & 93.125 & & 96.37 & 96.12 & 97.25 & 97.37 & 98.25 \\
\hline 9 & 1 & 92.00 & & 96.75 & 96.25 & 98.25 & 97.75 & 99.25 \\
\hline
\end{tabular}


Finally, to obtain the most similar faces in the database used, there are several distances measurement techniques are used. Also, a comparison has been done between the proposed system and PCA strategy; it shows that the presented algorithm performance is better than using the Eigenface technique alone using the three classifiers (ED, MD, and CD). In addition, it is found that the efficiency of the proposed schema is affected by the number of levels in DWT and the type of filter bank used.

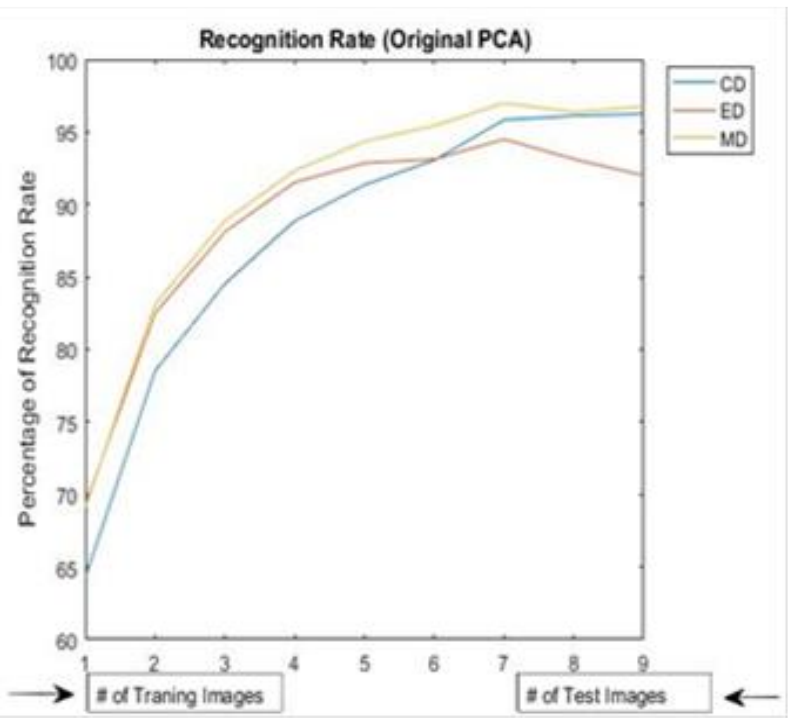

Fig (5) : Recognition rate for ORL database based PCA with distances classifier

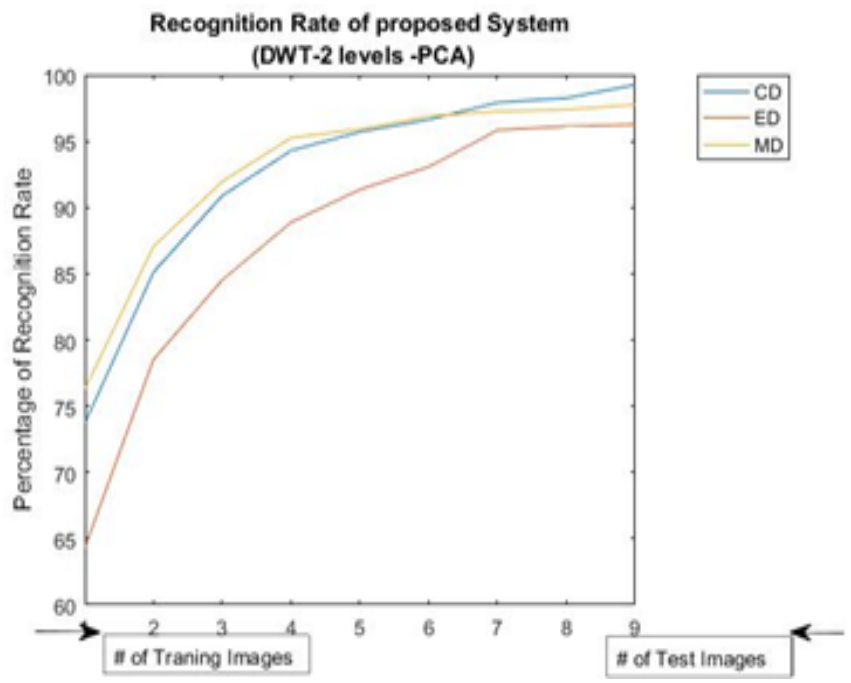

Fig (6) : Recognition rate for ORL database based proposed recognition system PCA and DWT with distances classifier
From the Fig. 5, show the Principal Component Analysis (PCA) using ORL database performance with the three distance measures (recognition classifiers). But in Fig. 6, show the accuracy of recognition rate of the proposed system which shows that the recognized users is improved. It is clear that the DWT (2 levels-bior5.5) with PCA gives better results with the three distance classifiers (Euclidian, Manhattan and Cosine), especially at the Cosine distance classifier, which gives highest accuracy results (above $99.25 \%$ recognition rate). So it is clearly seen from Table 2 and Fig. 5 and Fig. 6 that this technique of recognition is useful for identification closing set, and it is stable with recognition when the image samples of person are increased. Additionally, as shown in Table 3, a comparison between the most recent existing works available in the literature. The presented technique outperforms the state-of-the-art studies in terms of both the successful accuracy and database.

Table (3) : The Performance of state-of-the-art face recognition systems compared with proposed technique.

\begin{tabular}{|c|c|c|c|}
\hline $\begin{array}{c}\text { Face Recognition } \\
\text { Methodology }\end{array}$ & $\begin{array}{c}\text { Database/ } \\
\text { No. of Face } \\
\text { Samples }\end{array}$ & $\begin{array}{c}\text { Successful } \\
\text { Recognition } \\
\text { rate } \%\end{array}$ & Reference \\
\hline PCA, with DWT & ORL & $95.33 \%$ & $\begin{array}{c}\text { (Haider \& Arasi, } \\
\text { 2013) }\end{array}$ \\
\hline 3-level DWT- PCA & Yale & $93 \%$ & $\begin{array}{l}\text { (Abdulrahman, } \\
\text { Dambatta, } \\
\text { Muhammad, \& } \\
\text { Muhammad, } \\
\text { 2014) }\end{array}$ \\
\hline $\begin{array}{c}\text { Pre-Processing } \\
\text { technique with PCA }\end{array}$ & ORL & $97.5 \%$ & $\begin{array}{c}\text { (Barnouti N. H., } \\
\text { 2016) }\end{array}$ \\
\hline $\begin{array}{c}\text { Proposed: System } \\
\text { utilized } \\
\text { DWT+PCA with } \\
\text { CD classifier }\end{array}$ & ORL & 99.25 & \\
\hline
\end{tabular}

As it is clear from Table 3, the results obtained of the proposed method which is using DWT concatenated with PCA has the successful accuracy $99.25 \%$ and is compared with the recent three face recognition systems. Also, as it is seen by comparison, the 
recognition rate of the proposed method enhanced, which it is very important for human identification systems.

\section{CONCLUSION}

In this research, the main points are to enhance performance and accuracy of face recognition for human identification using different levels of DWT on ORL Database using MATLAB 2016a version, followed by feature extraction and dimension reduction using PCA model. The resulted test and train images finally measured with cosine distance, Euclidean distance, and city block distance measurements. The best face recognition results appear when comparing results of the proposed method with the original PCA model by using cosine distance measurement which is $99.25 \%$ recognition rate, which gives a powerful enhancement to the model with low time consumption. The proposed technique enhances the accuracy and recognition rate for human face identification under variant poses and illumination conditions. For the future work, the hybrid technique by mixing between DWT- Histogram Orientation Gradient (HOG) and PCA might merge the represented feature vectors. And this may improve the person recognition rate.

\section{References}

1.Abdulrahman, M., Dambatta, Y. G., Muhammad, A. S., \& Muhammad, A. S. (2014). Face Recognition Using Eigenface and Discrete Wavelet Transform. international Conference on Advances in Engineering and

Technology „,http:// dx.doi.org/10.15242/IIE.E0314004.

2.Bagherian, E., \& Rahmat, R. W. (2008). Facial feature extraction for face recognition: a review. IEEE.

3.Barnouti, N. H. (2016). Improve Face Recognition Rate Using Different Image Pre-Processing Techniques. American Journal of Engineering Research (AJER) -eISSN: 2320-0847 p-ISSN : 2320-0936, pp-46-53.

4.Barnouti, N. H., Mahmood, S. S., \& Matti, W. E. (2016). Face Recognition: A Literature Review. International Journal of Applied Information Systems (IJAIS) - ISSN :
2249-0868,Foundation of Computer Science FCS, New York, USA.

5.Gawande, M. P., \& Agrawal, D. G. (2014). Face recognition using PCA and different distance classifiers. Journal of Electronics and Communication Engineering (IOSR-JECE), PP 01-05.

6.Gumede, A., Viriri, S., \& Gwetu, M. (2017). Hybrid Component-based Face Recognition. IEEE.

7.Haider, A. S., \& Arasi, M. A. (2013). Face Recognition Based on PCA, DCT, DWT and Distance Measure. Egyptian Computer Science Journal Vol. 37 No. 5 September 2013 ISSN-1110-2586.

8.Kurmi, U. S., Agrawal, D., \& Baghel, R. K. (2014). Study of Different Face Recognition Algorithms and Challenges. International Journal of Engineering Research (ISSN:2319-6890)(online),2347-5013(print), Volume No.3, Issue No.2, pp : 112-115 01 Feb. 2014.

9.Mei, T. Y., Bo, T. J., Hua, C., \& Wei, L. (2010). A Study of Image Compression Technique. Fourth International Conference on Genetic and Evolutionary Computing (pp. 827-828). IEEE Xplore.

10.Nicholl, P., Ahmad, A., \& Amira, A. (2010). Optimal Discrete Wavelet Transform (DWT) Features for Face Recognition. IEEE.

Rao, M. K., Swamy, K. V., \& sheela, K. A. (2012). Face recognition using DWT and eigenvectors. IEEE.

11.Setyadi, A. D., Harsono, T., \& Wasista, S. (2015). Human Character Recognition Application Based on Facial Feature Using Face Detection. IEEE-International Electronics Symposium (IES).

12.Sodhi, K. S., \& Lal, M. (2013). FACE RECOGNITION USING PCA, LDA AND VARIOUS DISTANCE CLASSIFIERS. Journal of Global Research in Computer Science-ISSN-2229-371X.

13.Woods, J. W. (2012). Multidimensional Signal, Image, and Video Processing and Coding. United States of America: Academic Press is an imprint of Elsevier. 